\section{Ökostrom: keinesfalls überflüssig!}

\author{
Florian Wetzig fragte in der letzten Ausgabe, ob Ökostrom eine sinnvolle \\ Ergänzung zum Erneuerbare Energien Gesetz sei. Er sprach sich gegen den Kauf \\ von Ökostrom aus. Doch seine Kritik greift zu kurz. Hochwertige Ökostrom- \\ produkte schützen die Umwelt über die gesetzliche Förderung erneverbarer En- \\ ergien hinaus.
}

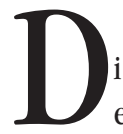

Von Veit Bürger Okostrommarktes ist angesichts eines gut funktionierenden Förderinstruments wie dem Erneuerbare Energien Gesetz (EEG) nicht neu. Im Mittelpunkt steht die Frage, wie das Zusammenspiel zwischen staatlichen Fördermechanismen und einem auf freiwilliger Nachfrage basierenden Ökostrommarkt synergetisch geregelt werden kann.

Florian Wetzig argumentiert vollkommen richtig, wenn er sagt, der Kauf von Ökostromprodukten bewirke nicht automatisch eine über das EEG hinausgehende Umweltentlastung. Viele Stromversorger bieten Ökostrom feil, der aus regenerativen Kraftwerken stammt, die der Anbieter schon lange in seinem Erzeugungsportfolio hält. In diesem Fall ist es offensichtlich, dass die Nachfrage nach einem solchen Produkt zu keiner zusätzlichen Umweltentlastung führt. Vielmehr kommt es lediglich zu einer Entmischung des Absatzportfolios eines Anbieters: Kunden, die bewusst nach Ökostrom fragen, erhalten den bestehenden erneuerbaren Anteil des Gesamtportfolios; alle anderen Kunden, die keine spezifische Ökopräferenz haben, erhalten den entmischen Rest.

\section{Zwei Anlagetypen}

Dementsprechend ist die Frage berechtigt, inwieweit der Ökostromhandel neben der Förderung durch das EEG zusätzliche Erzeugungspotenziale wecken kann. In der Bewertung unterscheidet der Autor zwischen zwei Anlagenkategorie: erstens EEG-fähige regenerative Kraftwerke (REG-Anlagen), die mit den Vergütungssätzen des EEG wirtschaftlich betrieben werden können. Zweitens REG-Anlagen, die nicht den Förderkriterien des EEG entsprechen beziehungsweise REG-Anlagen, die zwar in den Förderkatalog des EEG fallen, jedoch mit den EEG Vergütungssätzen nicht wirtschaftlich betrieben werden können.
Ökostromprodukte, die ausschließlich auf Anlagen der ersten Kategorie zurückgreifen, stellen lediglich eine Kostenverschiebung dar. Die Kosten, die bei Nutzung des EEG aufgrund des darin enthaltenen Umlagemechanismus im Normalfall die Allgemeinheit der Stromkunden tragen würde, werden den Ökostromkunden zugeordnet. Eine Erzeugung zusätzlicher regenerativer Kilowattstunden ist mit dem Kauf solcher Produkte nicht verbunden.

Bei der zweiten Anlagenkategorie zieht der Autor jedoch den falschen Schluss, wenn er argumentiert, dass dieser Anlagentypus durch den Ökostrommarkt generell nicht gefördert werden sollte, weil solche Anlagen entweder zu teuer oder ökologisch bedenklich seien:

- Nicht alle Anlagen, die durch das EEG nicht gefördert werden, sind ökologisch bedenklich. Allen voran ist hier die Zufeuerung von Biomasse in fossil befeuerten Kraftwerken zu nennen. Wird beispielsweise in einem Kohlekraftwerk ein Teil der Kohle durch Qualitäts-Biomasse ersetzt, die füllt, wird dadurch ein kostengünstiger Umweltschutzbeitrag geleistet.

- Darüber hinaus können Ökostromanbieter Anlagen im Ausland unterstïtzen, die dort keiner dem EEG entsprechenden Förderung unterliegen, sondern im Wettbewerb betrieben werden müssen. Da das EEG in Deutschland sehr gute Rahmenbedingungen für den Ausbau erneuerbarer Energien bietet, ist es für Ökostromprodukte die richtige Strategie, Neuanlagen im Ausland zu fördern, wo kein so effizientes Förderinstrument besteht. Aus Gründen der Transparenz sollten jedoch Stromanbieter ihre Kunden über die Herkunft des Stroms informieren.

- Auch die sogenannten Fondsmodelle haben selbst nach der aktuellen EEG-Novelle - ihre Berechtigung. Mit der Festlegung von fixen Vergütungssätzen zieht das EEG eine willkürliche Grenze für die Wirtschaftlichkeit von potenziellen Anlagenstandorten. Durch die Zahlung einer bedie Anforderungen der Biomasseverordnung er- grenzten Zusatzvergütung als Aufschlag zu dem EEG-Satz wird der Bereich der wirtschaftlichen Anlagenstandorte vergrößert.

EEG und Ökostrommarkt stellen demnach keine Gegensätze dar. Vielmehr können sich beide Instrumente hervorragend ergänzen. Natürlich ist bisher das EEG das effektivere Instrument für den REG-Ausbau.

\section{Label als Garant}

Damit der Ökostrommarkt das EEG sinnvoll ergänzt, bedarf es allerdings sorgfältig ausgearbeiteter Kriterien. Diese müssen sicherstellen, dass die freiwillige Ökostromnachfrage zu einer Ausweitung der regenerativen Stromerzeugung führt, die über den Anlagenbestand und den Wirkungsbereich des EEG hinausgeht. Dies stellt beispielsweise der Kriterienkatalog des ok-power Labels sicher (2). Um einen Anreiz zum Neubau von Ökostrom-Erzeugungsanlagen zu geben, muss jedes zertifizierte Produkt in jedem Kalenderjahr mindestens zu einem Drittel aus Strom aus REGNeuanlagen kommen. Neuanlagen sind dabei Anlagen, die nicht älter als sechs Jahre sind. Anlagen, die unter das EEG fallen, werden dabei - unabhängig von ihrem Alter - nicht als Neuanlagen anerkannt. Damit müssen die Anbieter der zertifizierten Produkte spätestens alle sechs Jahre neue Anlagen unter Vertrag nehmen, die nicht durch das EEG gefördert werden.

Durch solche Regelungen werden die Käufer von konventionellem Strom nicht von den Kosten des Umweltschutzes entlastet. Vielmehr beteiligen sie sich auch weiterhin über den EEG-Umlagemechanismus an diesen Kosten. Kunden, die jedoch mehr für die Umwelt tun wollen, steht mit dem Kauf eines zertifizierten Ökostromproduktes eine einfache Möglichkeit offen, dies zu tun. Eine Umkehrung des Verursacherprinzips ist damit nicht gegeben.

\section{Anmerkungen}

(1) Vgl. Wetzig, F.: Wo bleibt das Verursacherprinzip?

In: Ökologisches Wirtschaften 3-4/2004

(2) Informationen unter: www.ok-power.de

\section{Der Autor}

Veit Bürger ist wissenschaftlicher Mitarbeiter am Öko-Institut.

Kontakt: Öko-Institut, Binzengrün 34a

79114 Freiburg. Tel. 0761-4529559,

E-Mail: v.buerger@oeko.de 
(c) 20I0 Authors; licensee IÖW and oekom verlag. This is an article distributed under the terms of the Creative Commons Attribution Non-Commercial No Derivates License (http://creativecommons.org/licenses/by-nc-nd/3.o/), which permits unrestricted use, distribution, and reproduction in any medium, provided the original work is properly cited. 